\title{
THE TWO-DIMENSIONAL HARMONIC OSCILLATOR ON A NONCOMMUTATIVE SPACE WITH MINIMAL UNCERTAINTIES
}

\author{
SANJiB DEY*, ANDreas Fring \\ Department of Mathematical Science, City University London, Northampton Square, London EC1V OHB, UK \\ * corresponding author: sanjib.dey.1@city.ac.uk
}

\begin{abstract}
The two dimensional set of canonical relations giving rise to minimal uncertainties previously constructed from a q-deformed oscillator algebra is further investigated. We provide a representation for this algebra in terms of a flat noncommutative space and employ it to study the eigenvalue spectrum for the harmonic oscillator on this space. The perturbative expression for the eigenenergy indicates that the model might possess an exceptional point at which the spectrum becomes complex and its PT-symmetry is spontaneously broken.
\end{abstract}

KEYWORDS: noncommutative space, non-Hermitian operators, 2D-systems.

In [1] we demonstrated how canonical relations implying minimal uncertainties can be derived from a $q$-deformed oscillator algebra for the creation and annihilation operators $A_{i}^{\dagger}, A_{i}$

$$
\left.\begin{array}{rl}
A_{i} A_{j}^{\dagger}-q^{2 \delta_{i j}} A_{j}^{\dagger} A_{i} & =\delta_{i j}, \\
{\left[A_{i}^{\dagger}, A_{j}^{\dagger}\right]} & =0, \\
{\left[A_{i}, A_{j}\right]} & =0,
\end{array}\right\} \text { for } i, j=1,2,3 ; q \in \mathbb{R}
$$

as investigated for instance in [2] . Starting from the general Ansatz

$$
\begin{aligned}
& X=\hat{\kappa}_{1}\left(A_{1}^{\dagger}+A_{1}\right)+\hat{\kappa}_{2}\left(A_{2}^{\dagger}+A_{2}\right) \\
& +\hat{\kappa}_{3}\left(A_{3}^{\dagger}+A_{3}\right), \\
& Y=i \hat{\kappa}_{4}\left(A_{1}^{\dagger}-A_{1}\right)+i \hat{\kappa}_{5}\left(A_{2}^{\dagger}-A_{2}\right) \\
& +i \hat{\kappa}_{6}\left(A_{3}^{\dagger}-A_{3}\right), \\
& Z=\hat{\kappa}_{7}\left(A_{1}^{\dagger}+A_{1}\right)+\hat{\kappa}_{8}\left(A_{2}^{\dagger}+A_{2}\right) \\
& +\hat{\kappa}_{9}\left(A_{3}^{\dagger}+A_{3}\right), \\
& P_{x}=i \check{\kappa}_{10}\left(A_{1}^{\dagger}-A_{1}\right)+i \check{\kappa}_{11}\left(A_{2}^{\dagger}-A_{2}\right) \\
& +i \check{\kappa}_{12}\left(A_{3}^{\dagger}-A_{3}\right), \\
& P_{y}=\check{\kappa}_{13}\left(A_{1}^{\dagger}+A_{1}\right)+\check{\kappa}_{14}\left(A_{2}^{\dagger}+A_{2}\right) \\
& +\check{\kappa}_{15}\left(A_{3}^{\dagger}+A_{3}\right) \\
& P_{z}=i \check{\kappa}_{16}\left(A_{1}^{\dagger}-A_{1}\right)+i \check{\kappa}_{17}\left(A_{2}^{\dagger}-A_{2}\right) \\
& +i \check{\kappa}_{18}\left(A_{3}^{\dagger}-A_{3}\right) \text {, }
\end{aligned}
$$

with $\hat{\kappa}_{i}=\kappa_{i} \sqrt{\hbar /(m \omega)}$ for $i=1, \ldots, 9$ and $\check{\kappa}_{i}=$ $\kappa_{i} \sqrt{m \omega \hbar}$ for $i=10, \ldots, 18$ we constructed some particular solutions and investigated the harmonic oscillator on these spaces. Here we provide an additional two dimensional solution previously reported in [6]. Setting $\kappa_{3}=\kappa_{6}=\kappa_{7}=\kappa_{12}=\kappa_{15}=\kappa_{16}=\kappa_{17}=$ $\kappa_{18}=0$ in equations $(2 \mathrm{a}-2 \mathrm{ff})$, employing the constraints reported in [6] together with the subsequent nontrivial limit $q \rightarrow 1$, the deformed oscillator algebra

$$
\begin{aligned}
{[X, Y] } & =i \theta\left(1+\hat{\tau} Y^{2}\right), & {\left[P_{x}, P_{y}\right] } & =i \hat{\tau} \frac{\hbar^{2}}{\theta} Y^{2}, \\
{\left[X, P_{x}\right] } & =i \hbar\left(1+\hat{\tau} Y^{2}\right), & {\left[Y, P_{y}\right] } & =i \hbar\left(1+\hat{\tau} Y^{2}\right), \\
{\left[X, P_{y}\right] } & =0, & {\left[Y, P_{x}\right] } & =0,
\end{aligned}
$$

was obtained, with $\hat{\tau}=\tau m \omega / \hbar$ having the dimension of an inverse squared length. By the same reasoning as provided in [1, 5, 9], we find the minimal uncertainties

$$
\begin{aligned}
\Delta X_{\min } & =|\theta| \sqrt{\hat{\tau}+\hat{\tau}^{2}\langle Y\rangle_{\rho}^{2}}, \\
\Delta Y_{\min } & =0, \\
\Delta\left(P_{x}\right)_{\min } & =0, \\
\Delta\left(P_{y}\right)_{\min } & =\hbar \sqrt{\hat{\tau}+\hat{\tau}^{2}\langle Y\rangle_{\rho}^{2}},
\end{aligned}
$$

where $\langle.\rangle_{\rho}$ denotes the inner product on a Hilbert space with metric $\rho$ in which the operators $X, Y, P_{x}$ and $P_{y}$ are Hermitian. So far no representation for the two dimensional algebra (3) has been provided. Here we find that it can be represented by

$$
\begin{aligned}
X & =x_{0}+\hat{\tau} y_{0}^{2} x_{0}, \\
Y & =y_{0}, \\
P_{x} & =p_{x_{0}}, \\
P_{y} & =p_{y_{0}}-\hat{\tau} \frac{\hbar}{\theta} y_{0}^{2} x_{0},
\end{aligned}
$$

where $x_{0}, y_{0}, p_{x_{0}}, p_{y_{0}}$ satisfy the common commutation relations for the flat noncommutative space

$$
\begin{array}{rrrrl}
{\left[x_{0}, y_{0}\right]} & =i \theta, & {\left[x_{0}, p_{x_{0}}\right]=i \hbar,} & {\left[x_{0}, p_{y_{0}}\right]=0} \\
{\left[p_{x_{0}}, p_{y_{0}}\right]=0,} & {\left[y_{0}, p_{y_{0}}\right]=i \hbar,} & {\left[y_{0}, p_{x_{0}}\right]=0} \\
\text { for } \theta & \in \mathbb{R} .
\end{array}
$$

Clearly there exist many more solutions that one may construct in this systematic manner from the 
Ansatz (2a) - 2f , which will not be our concern here. Instead we will study a concrete model, i.e. the twodimensional harmonic oscillator on the noncommutative space described by algebra (3). Using representation (5), the corresponding Hamiltonian reads

$$
\begin{aligned}
& H_{\text {ncho }}^{2 D}=\frac{1}{2 m}\left(P_{x}^{2}+P_{y}^{2}\right)+\frac{m \omega^{2}}{2}\left(X^{2}+Y^{2}\right) \\
& =H_{\text {fncho }}^{2 D}+\frac{\hat{\tau}}{2}\left[m \omega^{2}\left\{y_{0}^{2} x_{0}, x_{0}\right\}-\frac{\hbar}{m \theta}\left\{y_{0}^{2} x_{0}, p_{y_{0}}\right\}\right] \\
& \quad+\frac{\hat{\tau}^{2}}{2}\left[m \omega^{2}+\frac{\hbar^{2}}{m \theta^{2}}\right] y_{0}^{2} x_{0} y_{0}^{2} x_{0},
\end{aligned}
$$

where we used the standard notation for the anticommutator $\{A, B\}:=A B+B A$. Evidently this Hamiltonian is non-Hermitian with regard to the standard inner product, but respects an antilinear symme$\operatorname{try} \mathcal{P} \mathcal{T}_{ \pm}: x_{0} \rightarrow \pm x_{0}, y_{0} \rightarrow \mp y_{0}, p_{x_{0}} \rightarrow \mp p_{x_{0}}, p_{y_{0}} \rightarrow$ $\pm p_{y_{0}}, i \rightarrow-i$. This suggests that its eigenvalue spectrum might be real, or at least real in parts [10-12]. Let us now investigate the spectrum perturbatively around the solution of the standard harmonic oscillator. In order to perform such a computation we need to convert flat noncommutative space into the standard canonical variable $x_{s}, y_{s}, p_{x_{s}}$ and $p_{y_{s}}$. This is achieved by means of a so-called Bopp-shift $x_{0} \rightarrow x_{s}-\frac{\theta}{\hbar} p_{y_{s}}, y_{0} \rightarrow y_{s}, p_{x_{0}} \rightarrow p_{x_{s}}$ and $p_{y_{0}} \rightarrow p_{y_{s}}$. The Hamiltonian in (7) then acquires the form

$$
\begin{aligned}
& H_{\mathrm{ncho}}^{2 D}=H_{\mathrm{ho}}^{2 D}+\frac{m \theta^{2} \omega^{2}}{2 \hbar^{2}} p_{y_{s}}^{2}-\frac{m \theta \omega^{2}}{2 \hbar}\left\{x_{s}, p_{y_{s}}\right\} \\
& +\frac{\hat{\tau}}{2}\left[m \omega^{2}\left\{y_{s}^{2} x_{s}, x_{s}\right\}-\frac{\hbar}{m \theta}\left\{y_{s}^{2} x_{s}, p_{y_{s}}\right\}\right] \\
& +\frac{\hat{\tau}}{2}\left[\left(\frac{1}{m}+\frac{m \theta^{2} \omega^{2}}{\hbar^{2}}\right)\left\{y_{s}^{2} p_{y_{s}}, p_{y_{s}}\right\}\right. \\
& \left.+\frac{\hat{\tau}^{2}}{2}\left[\frac{m \theta \omega^{2}}{\hbar}+\frac{\hbar}{m \theta}\right]\left(\left\{y_{s}^{2} p_{y_{s}}, x_{s}\right\}+\left\{y_{s}^{2} x_{y_{s}}, p_{y_{s}}\right\}\right)\right] \\
& +\frac{\left.\hat{\tau}^{2} x_{s}+y_{s}^{2} x_{s} y_{s}^{2} p_{y_{s}}\right)}{2}\left[\frac{1}{m}+\frac{m \theta^{2} \omega^{2}}{\hbar^{2}}\right] y_{s}^{2} p_{y_{s}} y_{s}^{2} p_{y_{s}} \\
& +\frac{\hat{\tau}^{2}}{2}\left[m \omega^{2}+\frac{\hbar^{2}}{m \theta^{2}}\right] y_{s}^{2} x_{s} y_{s}^{2} x_{s} \\
& =H_{\mathrm{ho}}^{2 D}\left(x_{s}, y_{s}, p_{x_{s}}, p_{y_{s}}\right)+H_{\mathrm{nc}}^{2 D}\left(x_{s}, y_{s}, p_{x_{s}}, p_{y_{s}}\right) .
\end{aligned}
$$

In this formulation we may now proceed to expand perturbatively around the standard two dimensional Fock space harmonic oscillator solution with normalized eigenstates

$$
\begin{aligned}
\left|n_{1} n_{2}\right\rangle & =\frac{\left(a_{1}^{\dagger}\right)^{n_{1}}\left(a_{2}^{\dagger}\right)^{n_{2}}}{\sqrt{n_{1} ! n_{2} !}}|00\rangle, \\
a_{i}^{\dagger}\left|n_{1} n_{2}\right\rangle & =\sqrt{n_{i}+1}\left|\left(n_{1}+\delta_{i 1}\right)\left(n_{2}+\delta_{i 2}\right)\right\rangle, \\
a_{i}|00\rangle & =0, \\
a_{i}\left|n_{1} n_{2}\right\rangle & =\sqrt{n_{i}}\left|\left(n_{1}-\delta_{i 1}\right)\left(n_{2}-\delta_{i 2}\right)\right\rangle,
\end{aligned}
$$

for $i=1,2$, such that $H_{\text {ho }}^{2 D}|n l\rangle=E_{n l}^{(0)}|n l\rangle$. The energy eigenvalues for the Hamiltonian $H_{\mathrm{ncho}}^{2 D}$ then result to

$$
\begin{aligned}
& E_{n l}^{(p)}=E_{n l}^{(0)}+E_{n l}^{(1)}+E_{n l}^{(2)}+\mathcal{O}\left(\tau^{2}\right) \\
& =E_{n l}^{(0)}+\left\langle n l\left|H_{\mathrm{nc}}^{2 D}\right| n l\right\rangle \\
& \quad+\sum_{p, q \neq n+l=p+q} \frac{\left\langle n l\left|H_{\mathrm{nc}}^{2 D}\right| p q\right\rangle\left\langle p q\left|H_{\mathrm{nc}}^{2 D}\right| n l\right\rangle}{E_{n l}^{(0)}-E_{p q}^{(0)}}+\mathcal{O}\left(\tau^{2}\right) \\
& =\omega \hbar(n+l+1)+\frac{1}{16} \hbar \omega \Omega[2 n-(2 l+1) \Omega+10 l+6] \\
& +\frac{1}{8} \hbar \tau \omega\left[\Omega\left(8 n l+4 n+6 l^{2}+10 l+5\right)\right. \\
& \left.\quad+10 n l+5 n+5 l^{2}+10 l+5\right]+\mathcal{O}\left(\tau^{2}\right),
\end{aligned}
$$

where $\Omega=m^{2} \theta^{2} \omega^{2} / \hbar^{2}$. We note the minus sign in one of the terms, which might be an indication for the existence of an exceptional point [13, 14 in the spectrum. Naturally it would be very interesting to obtain a more precise expression for the eigenenergies, but nonetheless as has turned out to be very useful in the one dimensional setting [15] the first order approximations is very useful for the computation of coherent states [16].

\section{ACKNOWLEDGEMENTS}

S.D. is supported by a City University Research Fellowship.

\section{REFERENCES}

[1] S. Dey, A. Fring, and L. Gouba, PT-symmetric noncommutative spaces with minimal volume uncertainty relations, J. Phys. A: Math. Theor. 45 (2012) 385302

[2] L. C. Biedenham, The quantum group group $S U(2)_{q}$ and a q-analogue of the boson operators, J. Phys. A22, L873-L878 (1989).

[3] A. J. Macfarlane, On q-analogues of the quantum harmonic oscillator and the quantum group $S U(2)_{q}, \mathrm{~J}$. Phys. A22, 4581-4588 (1989).

[4] C.-P. Su and H.-C. Fu, The q-deformed boson realisation of the quantum group $S U(n)_{q}$ and its representations, J. Phys. A22, L983-L986 (1989).

[5] B. Bagchi and A. Fring, Minimal length in Quantum Mechanics and non-Hermitian Hamiltonian systems, Phys. Lett. A373, 4307-4310 (2009).

[6] A. Fring, L. Gouba, and B. Bagchi, Minimal areas from q-deformed oscillator algebras, J. Phys. A43, 425202 (2010).

[7] A. Kempf, Uncertainty relation in quantum mechanics with quantum group symmetry, J. Math. Phys. 35, 4483-4496 (1994).

[8] A. Kempf, G. Mangano, and R. B. Mann, Hilbert space representation of the minimal length uncertainty relation, Phys. Rev. D52, 1108-1118 (1995).

[9] A. Fring, L. Gouba, and F. G. Scholtz, Strings from dynamical noncommutative space-time, J. Phys. A43, 345401(10) (2010)

[10] C. M. Bender and S. Boettcher, Real Spectra in Non-Hermitian Hamiltonians Having PT Symmetry, Phys. Rev. Lett. 80, 5243-5246 (1998). 
[11] A. Mostafazadeh, Pseudo-Hermiticity versus PT symmetry: The necessary condition for the reality of the spectrum of a non-Hermitian Hamiltonian, J. Maths. Phys. 43, 202-212 (2002).

[12] C. M. Bender, Making sense of non-Hermitian Hamiltonians, Rept. Prog. Phys. 70, 947-1018 (2007).

[13] C. M. Bender and T. T. Wu, Anharmonic Oscillator, Phys. Rev. 184, 1231-1260 (1969).
[14] T. Kato, Perturbation Theory for Linear Operators, (Springer, Berlin) (1966).

[15] S. Dey, A. Fring, L. Gouba, and P. G. Castro, Timedependent $q$-deformed coherent states for generalised uncertainty relations, Phys. Rev. D 87, 084033 (2013).

[16] S. Dey and A. Fring, Squeezed coherent states for noncommutative spaces with minimal length uncertainity relations, Phys. Rev D 86, 064038 (2012). 